\title{
Un retour vers le futur avec Lucien Matrat, le père fondateur des Relations Publiques Européennes
}

Hélène Arzeno-Martin

\section{OpenEdition}

1 Journals

Édition électronique

URL : http://journals.openedition.org/communicationorganisation/1672

DOI : 10.4000/communicationorganisation. 1672

ISSN : $1775-3546$

Éditeur

Presses universitaires de Bordeaux

Édition imprimée

Date de publication : 1 novembre 1993

ISSN : 1168-5549

Référence électronique

Hélène Arzeno-Martin, « Un retour vers le futur avec Lucien Matrat, le père fondateur des Relations Publiques Européennes », Communication et organisation [En ligne], 4 | 1993, mis en ligne le 26 mars 2012, consulté le 19 avril 2019. URL : http://journals.openedition.org/

communicationorganisation/1672 ; DOI : 10.4000/communicationorganisation.1672

Ce document a été généré automatiquement le 19 avril 2019

(c) Presses universitaires de Bordeaux 


\title{
Un retour vers le futur avec Lucien Matrat, le père fondateur des Relations Publiques Européennes
}

\author{
Hélène Arzeno-Martin
}

1 Dans les instances professionnelles des Relations Publiques, à l'AFREP (Association Française des Relations Publiques), comme à la CERP (Confédération Européenne des Relations Publiques) ou à l'IPRA (International Public Relation Association), régulièrement, quand les réflexions sur l'avenir de la profession se font plus profondes, quand on commence à associer des termes comme efficacité et éthique, un nom surgit : Lucien Matrat. Et si on demande "Qui est Lucien Matrat? ", il se trouve toujours quelqu'un pour lancer, presque offusqué de cette ignorance, «mais c'est le père fondateur des Relations Publiques Européennes!». Une pareille dénomination ne pouvait laisser indifférent et le $\mathrm{GRE} / \mathrm{CO}$ a voulu aller à la rencontre de ce personnage à la pensée novatrice.

2 C'est dans la banlieue parisienne que Lucien Matrat jouit d'une retraite bien méritée, troublée malheureusement par quelques problèmes de santé, ce qui nous a conduit à une brève interview téléphonique nous renvoyant essentiellement à ses écrits issus des nombreuses conférences qu'il a pu donner à travers l'Europe depuis plus de quarante ans. Nous en avons extrait l'essentiel d'une pensée qui témoigne de concepts d'une actualité étonnante.

\section{Déjà, en 1951, à Bordeaux}

3 Plus de 40 ans! C'est en 1951, à la Faculté de Droit de Bordeaux, au cours d'une conférence sur «Les responsabilités sociales de l'entreprise », que Lucien Matrat aborde les grandes lignes de tout son discours futur sur lequel s'édifieront les textes régissant la profession des relations publiques à l'échelle Européenne. Écoutons-le...

«...) La responsabilité sociale est celle qui nous oblige à répondre de nos actes ou de nos carences susceptibles de porter préjudice à l'harmonie de la société, étant 
entendu que cette harmonie repose sur l'ensemble des individus, des groupes et des classes qui constituent cette société et sur leur adhésion aux principes qui régissent son fonctionnement. (...) Admettre que l'entreprise doit répondre de ses actions et de ses carences (...) c'est admettre que l'entreprise doit jouer, au sein de celle-ci, un rôle nouveau: le rôle d'une cellule sociale dont le but est précisément d'aider au développement de la société. Si l'on accepte cette définition, on accepte du même coup d'assigner à l'entreprise, en plus de son rôle économique traditionnel, un but social, et on l'engage dès lors dans une voie nouvelle (...).

Le fait d'avoir considéré l'entreprise exclusivement comme une cellule économique aboutit à des impasses. Impasse sur le plan des activités productives (...), impasse aussi sur le plan de la société où l'harmonie entre les individus, entre les groupes, entre les classes est rompue (...). Les responsables de l'entreprise, qui l'ont considérée exclusivement sous son angle économique et qui ont cru que son efficacité était uniquement liée au progrès technique, à la rationalisation des méthodes de travail, n'ont-ils pas oublié «l'homme »?

(...) L'entreprise, par son développement, par la concentration industrielle qu'elle a suscitée, a déraciné l'homme de ses groupes primaires, de la civilisation rurale, pour le transplanter dans une civilisation urbaine. Elle l'a implanté dans des groupes d'une ampleur telle que les relations directes entre les membres du groupe ne sont plus possibles. (...) A l'autorité s'est substitué le commandement qui appelle trop souvent l'obéissance passive, voire la résistance (...). L'environnement humain de l'homme lui devient étranger: il n'a pas le sentiment de participer à ce qui conditionne sa vie collective.

\section{Recréer le sentiment d'interdépendance}

«(...) L'interdépendance qui existe entre les membres d'un groupe constitue une garantie de la cohésion de ce groupe, mais encore faut-il que cette interdépendance soit reconnue (...). En oubliant de dégager et de mettre en valeur ces facteurs d'interdépendance afin de les rendre évidents et de leur permettre de jouer leur rôle de lien social; en négligeant depuis toujours de justifier son existence, de montrer les services qu'elle apporte à la collectivité, l'entreprise commet non seulement une erreur à l'égard de l'opinion publique mais elle met en danger l'existence même du système de libre entreprise sur lequel est fondée notre société. (...) L'entreprise, à la recherche de l'efficacité, n'a-t-elle pas négligé cet aspect humain du problème qui se posait à elle, et cette omission est-elle vraiment sans danger pour l'harmonie de la société ? Là encore, sa responsabilité sociale ne se trouve-t-elle pas à nouveau engagée ? Quelque intéressants qu'aient été à ce sujet les travaux de certains sociologues français, il a fallu attendre que le mouvement qu'ils ont inspiré aux Etats-Unis nous revienne en France sous le vocable de " public relations ", pour qu'on commence à évoquer souvent, sinon admettre, le rôle et la responsabilité sociale de l'entreprise, tant vis-à-vis des hommes qui la constituent que vis-à-vis des groupes au milieu desquels elle vit.

(...) Les Américains se sont rendu compte que l'efficacité de l'entreprise était en fait très directement dépendante de sa structure sociale, et qu'en matière de production, l'homme, en définitive, restait le facteur déterminant. Ils ont remarqué que plus l'entreprise grossissait, plus elle arrivait, à s'abstraire du milieu au sein du quelle elle exerçait son activité et que, de ce fait, son développement se trouvait souvent freiné par une opinion publique mal informée (...). Par une information objective et permanente, l'entreprise peut rompre, sur le plan extérieur, cet espèce d'isolement aussi préjudiciable à son intérêt qu'à la création de relations confiantes entre les membres de la société.

4 Par l'information extérieure qui permet au public de savoir ce que fait l'entreprise, comment elle le fait, pourquoi elle le fait, en bref, de connaître ses projets et ses activités 
en tant qu'employeur, producteur, distributeur, gérant de capitaux, l'entreprise s'acquitte également d'une de ses responsabilités sociales.

\section{Des responsabilités vis-à-vis du personnel}

«Nous arrivons maintenant à un des points les plus importants -souligne Lucien Matrat -, aux devoirs que la responsabilité sociale de l'entreprise lui crée vis-à-vis de son personnel considéré non pas comme un personnel qui a des droits, mais comme un personnel qui a des besoins (...). Il s'agit de savoir si on va chercher à modifier la nature des hommes pour qu'ils soient satisfaits de l'entreprise ou si l'on va modifier le sens et la conception de l'entreprise pour qu'elle apporte, ou du moins qu'elle tente d'apporter, à ces hommes, ce que leur nature réclame.

(...) Si, sur le plan des machines, le carburant est indispensable, il n'est pas suffisant, il faut aussi un lubrifiant. L'homme, cette machine complexe, requiert pour «tourner", autre chose que le «carburant-salaire». Il a d'abord besoin d'être considéré comme un homme, pour s'obliger à en être un. Il a en lui le besoin de connaître, de comprendre, de croire, d'aimer et, inversement, le besoin de faire connaître, d'être compris, d'être cru et d'être aimé. Il a enfin le désir de s'intégrer à un ordre social et d'y progresser.

5 La solution tient en peu de mots : information, formation, promotion, intéressement et sécurité de l'emploi. Des mots-clefs qui constituent un programme par lequel l'entreprise peut lutter contre le manque d'attrait pour un travail dont on voit mal le sens, contre les préjugés, les idées fausses, contre les barrières de classe, contre le manque d'espoir et les complexes de frustration.

(...) Il est indispensable de concentrer les problèmes d'ordres sociaux entre les mains d'une personne: le responsable des relations humaines (dont) les responsabilités ont une importance au moins égale aux autres responsabilités traditionnelles, y compris l'économique et la financière.

\section{Vers un « bilan social »}

«L'entreprise, considérée comme cellule économique, rend compte par la publication d'un bilan financier. Pourquoi, si l'on admet qu'elle est aussi une cellule sociale et qu'elle a des responsabilités, ne devrait-elle pas aussi rendre compte de la façon dont elle s'est acquittée de ces responsabilités par la publication d'un "bilan social»? Ce bilan montrerait ce qu'elle a fait sur le plan de l'information, de la formation, de la promotion ouvrière, de l'intéressement, de la sécurité de l'emploi, en d'autres termes de ses réalisations sociales et de ses actions pour améliorer les relations humaines à l'intérieur et à l'extérieur de l'entreprise. "

Ainsi, il y a plus de quarante ans, Lucien Matrat, en précurseur, a su mettre en évidence l'intérêt des relations sociales, et par voie de conséquence, d'un bilan social insistant en conclusion de sa conférence sur la finalité de notre société « qui ne peut être qu'humaine car nous ne sommes ni une société de capitaux, ni une société de machines, mais une société d'hommes. »

\section{5 ans plus tard, le Code d'Athènes}

7 Ce professionnel des Relations Publiques qu'est Lucien Matrat -dès l'après-guerre il est directeur des Relations Extérieures d'un "pétrolier», Elf Aquitaine -, continue sa réflexion sur les responsabilités sociales de l'entreprise et sur les besoins spécifiques de 
l'homme social, entre autres sur ses besoins d'information, de communication, de dialogue et de participation.

Président Fondateur du « Centre Européen des Relations Publiques », le CERP, association regroupant les principales associations de relations publiques de toute l'Europe, Lucien Matrat est au cœur des discussions qui aboutissent, lors de l'Assemblée Générale qui s'est tenue à Athènes en mai 1965, à l'élaboration d'un Code d'Ethique International des Relations Publiques, dit LE CODE D'ATHENES, toujours en vigueur à l'heure actuelle, seulement réactualisé à Lisbonne au cours de deux assemblées de la CERP.

On retrouve la marque de Lucien Matrat dans ce Code où les Associations membres de la CERP, se référant à la Déclaration Universelle des Droits de l'Homme, considèrent que l'homme, à côté de ses droits a des besoins (...), que les praticiens des Relations Publiques peuvent contribuer à satisfaire ces besoins intellectuels, moraux et sociaux; elles considèrent également que l'utilisation des techniques qui permettent d'entrer simultanément en contact avec des millions d'individus, donnent aux praticiens des Relations Publiques un pouvoir qu'il est nécessaire de limiter par le respect d'une stricte morale et se donnent ainsi pour Charte morale les principes d'un Code d'Ethique, le Code d'Athènes.

\section{5 : une Doctrine Européenne}

L'analyse des besoins spécifiques de l'homme social est la base de la "Doctrine Européenne des Relations Publiques » dont l'objet est de créer les conditions facilitant la satisfaction des besoins spécifiques de l'homme social. Cette Doctrine Européenne, « condition du dialogue et de la participation » fut élaborée, elle aussi, en 1966, par Lucien Matrat, toujours président de la CERP.

11 Devenue Confédération Européenne des Relations Publiques, la CERP rediffusa cette Doctrine à l'occasion de son premier congrès européen organisé en mai 1984, à Bruxelles, le document ayant été réédité par la Fédération Française des Relations Publiques, la FFRP, regroupement des associations françaises de relations publiques.

12 Lucien Matrat, depuis sa conférence de Bordeaux, en 1951, a eu le temps de peaufiner sa pensée. Reprenant sa notion d'HOMME SOCIAL, il en analyse les besoins dans l'optique des Relations Publiques, en distinguant trois personnages : la personne, le partenaire et le fabricant d'opinion, chacun se caractérisant par des besoins propres.

«La PERSONNE a un besoin de dignité. Le Code d'Athènes impose aux professionnels des relations publiques le respect de ces impératifs moraux. »

«Le PARTENAIRE a un besoin essentiel : participer. (...) La participation, commun dénominateur de l'épanouissement de l'homme et de la réussite de toute œuvre collective constitue la motivation et la justification sociale et économique de la Doctrine Européenne. Il s'agit donc, pour les relations publiques, de contribuer à réunir, au sein de l'entreprise, les conditions qui font que chaque partenaire se sente CONCERNE, RESPONSABLE, SOLIDAIRE, CONSIDERE.

Ces quatre objectifs sont les repères qui doivent guider l'entreprise (...) dans le choix des moyens et des méthodes à mettre en œuvre, tant sur le plan de l'organisation que sur celui de l'information et de la communication; plan dont on peut regrouper les actions autour de trois grands axes : connaissance, intégration et participation. (...)

LE FABRICANT D'OPINION : l'homme social (...) échange des opinions, des jugements, des idées, c'est-à -dire la matière première avec laquelle se fabrique 
l'opinion publique. Il ne peut exercer cette fonction de fabricant d'opinion que s'il reçoit les informations qui lui permettent de connaître, de comprendre, de juger, de s'exprimer et de communiquer.

L'information fournie par les relations publiques doit répondre à quatre critères : s'insérer dans le dialogue (...), être comprise (...), être acceptable et assimilable (...), respecter une éthique rigoureuse (...). »

\section{Ni publicité, ni propagande}

13 La Doctrine Européenne des Relations Publiques permet de ne plus confondre les relations publiques avec la publicité (stratégie du désir qu'on déclenche) ou la propagande (stratégie du conditionnement qu'on réalise). Les relations publiques sont la stratégie de la confiance qu'on mérite. En outre, comme le précise Lucien Matrat en conclusion, cette doctrine «contribue à la nécessaire conciliation des impératifs d'une organisation collective, imposée par les progrès techniques et l'impérieux devoir que nous avons tous de sauvegarder nos valeurs de civilisation fondées sur le respect de la personne. »

\section{Quel avenir pour les relations publiques?}

C'est en avril 1969, lors d'une réunion commune de la CERP et de l'IPRA à Dublin, que Lucien Matrat évoque le futur de cette profession: au sortir de la dernière guerre, «lorsque l'expression « relations publiques » commença à être utilisée sur notre vieux continent, le contenu de ces deux mots était assez imprécis. (...) Il existait alors un commun dénominateur qui était essentiellement, la recherche de la notoriété, l'un des objectifs de la publicité également. L'homme de R.P. devait être capable de concevoir l'astucieux événement ou l'adroite présentation permettant d'inonder la presse de communiqués. Les publicitaires s'élevèrent contre ces «squatters » et la presse condamna cette publicité de contrebande.»

Qu'en est-il 20 ans plus tard, c'est à dire en 1969, lorsque Lucien Matrat s'adresse aux membres de la CERP et de l'IPRA? «Les relations publiques s'emploient, trop souvent encore, à résoudre, à leur manière, des problèmes qui relèvent de la promotion des ventes ou de la publicité. La recherche de la notoriété, continuant à être pour beaucoup le but essentiel des relations publiques, fait souvent penser que la presse est le seul support. (...) L'objectif des relations publiques est d'établir et de maintenir des relations confiantes avec l'ensemble des publics de l'entreprise, la presse étant considérée comme un public et non pas comme un support.»

Lucien Matrat insiste, rappelons que nous sommes en 1969: «je prétends, si on veut sortir les relations publiques de l'ornière dans laquelle des «champions de l'action-àtout-prix » les maintiennent, qu'il faut d'abord définir clairement les objectifs et le domaine spécifique de la fonction relations publiques (...). Quatre faits caractérisent l'évolution de nos sociétés: tout d'abord, la rapidité toujours croissante des progrès scientifiques et techniques qui modifient le milieu de vie des hommes au rythme de véritables mutations (...), d'où la nécessité d'une nouvelle fonction sociale ayant pour objet de réduire les oppositions entre l'individu et son environnement.

17 (...) Le deuxième fait ce sont les formes nouvelles que prennent les phénomènes de socialisation et leurs répercussions sur les conditions du travail. Ces modifications posent de difficiles problèmes d'intégration et nuisent à la cohésion, (...) la sur spécialisation des 
tâches fait perdre au travail toute signification (...) d'où la nécessité d'informer, de communiquer si l'on veut que l'homme donne un sens à son effort. Seule une véritable science de la communication, fondée entre autres sur la psychologie sociale, peut contribuer à résoudre ce problème.

Le troisième fait est l'évolution des besoins des hommes et donc celle de leurs motivations au travail. C'est dans la participation qui fait de lui un partenaire du groupe que l'homme trouve un aliment à ces besoins du type être et paraître, condition de son épanouissement.

Quatrième fait auquel se réfère Lucien Matrat, l'évolution de l'environnement socioéconomique de l'entreprise et son influence sur sa marche et son développement. (...) Il ne s'agit pas d'adapter des produits ou des prestations économiques eux exigences du marché, c'est le rôle du marketing. Il s'agit de favoriser un meilleur ajustement entre les prestations de l'entreprise considérée sous son angle institutionnel, et les exigences des publics concernés. Là encore, un problème de communication, puisqu'il s'agit de maintenir, par le dialogue, des relations confiantes entre l'entreprise et son environnement (...).

\section{Une nouvelle fonction}

\section{Les relations publiques}

20 Eclairer le groupe, l'aider à adopter une politique conforme aux intérêts en présence, évitant ainsi des antagonismes, est une fonction sociale qui, par l'information, la communication, l'explication, permettra à l'homme de connaître et de comprendre pour participer et s'intégrer. Cette fonction sociale, indispensable à l'équilibre de l'homme, au progrès des groupes, à l'harmonie de la société ne serait-elle pas là, précisément, les domaines spécifiques de l'art et des techniques des relations publiques?

(...) Il faut donc que les relations publiques soient une véritable science de la communication, fondée sur la connaissance des sciences humaines; il faut aussi qu'elles soient une discipline sociale fondée, elle, sur une éthique rigoureuse; il faut enfin que leur méthodologie permette d'aborder les problèmes que posent la vie et le développement de tout groupe. Car ce sont ces trois points, une science de la communication, une discipline sociale et une méthodologie, qui manquent le plus tant à la société qu'à nos entreprises pour résoudre les problèmes nouveaux qui se posent à elles."

22 Monsieur Matrat, vous disiez cela il y a 25 ans à des professionnels de la communication. Vous traciez l'avenir des relations publiques et vous pensiez sûrement, à l'époque, que les années 90 verraient l'épanouissement de ces relations que vous qualifiez souvent de sociales.

Mais le problème reste entier. Tout ce que vous proposiez est malheureusement resté «lettre morte» la plupart du temps. L'aggravation du chômage brouille aujourd'hui toutes les données. Mais vous relire reste un baume bienfaisant : et si on essayait quand même? Et si on essayait de faire comprendre votre message à tous ces étudiants qui veulent entrer dans le métier faute d'avoir pu le donner aux générations qui les précèdent encore? 


\section{AUTEUR}

\section{HÉLÈNE ARZENO-MARTIN}

Responsable de la Formation Continue en Communication à l'IUT de Bordeaux Membre CERP et IPRA 